\title{
A ROLE OF INTERMEDIATE CHARGE STATE IN THE DX CENTER PHOTOIONISATION IN $\mathrm{Al}_{x} \mathrm{Ga}_{1-x} \mathrm{As}: \mathrm{Te}$
}

\author{
P. KACZOR AND Z.R. ŻYTKIEWICZ \\ Institute of Physics, Polish Academy of Sciences \\ Al. Lotników 32/46, 02-668 Warszawa, Poland
}

\begin{abstract}
Detailed studies of the DX center absorption are presented. These studies performed on thick AlGaAs:Te layers, give a strong indication for the influence of the intermediate charge state on the DX center photoionisation.

PACS numbers: 71.55.Eq
\end{abstract}

\section{Introduction}

Recently there has been presented the strong theoretical [1] and experimental $[2,3]$ evidence in favor of an interstitial-substitutional defect reaction governing the metastable behavior of the DX center. In this reaction, the DX center, forming a negative- $U$ system, binds two electrons according to the following scheme:

$$
D^{+}+2 \mathrm{e}^{-} \rightarrow D^{0}+\mathrm{e}^{-} \rightarrow D^{-}
$$

The single-charged, unstable, intermediate charge state $D^{0}$ is assumed to play an essential role in capture and emission of electrons by the DX center [2].

In this work we report the detailed study of the DX center ionisation absorption. We explain an anomaly in the kinetics of the photoionisation absorption to be due to the DX intermediate charge state photoionisation.

\section{Experimental}

The samples used in this experiment were thick (100-200 $\mu \mathrm{m})$, very homogeneous in composition liquid phase electroepitaxy (LPEE) layers [4]. Three alloy compositions were studied: $x=0.27, x=0.33$, and $x=0.41$. The free carrier concentration was $n=5 \times 10^{17} \mathrm{~cm}^{-1}$ with exception of the $x=0.41$ sample where it amounted $n=2 \times 10^{18} \mathrm{~cm}^{-1}$. All measurements were done with rapid scan Bomem DA3 Fourier transform spectrometer in the wave number range from 6500 to $11000 \mathrm{~cm}^{-1}$ at temperatures below $50 \mathrm{~K}$. 


\section{Results and discussion}

In absorption measurements a broad band around $1.2 \mathrm{eV}\left(c a .10000 \mathrm{~cm}^{-1}\right)$ was observed [5], which was similar to that studied by photocapacitance methods [2,6] (Fig. 1). The low energy tail of this absoption merges (for composition

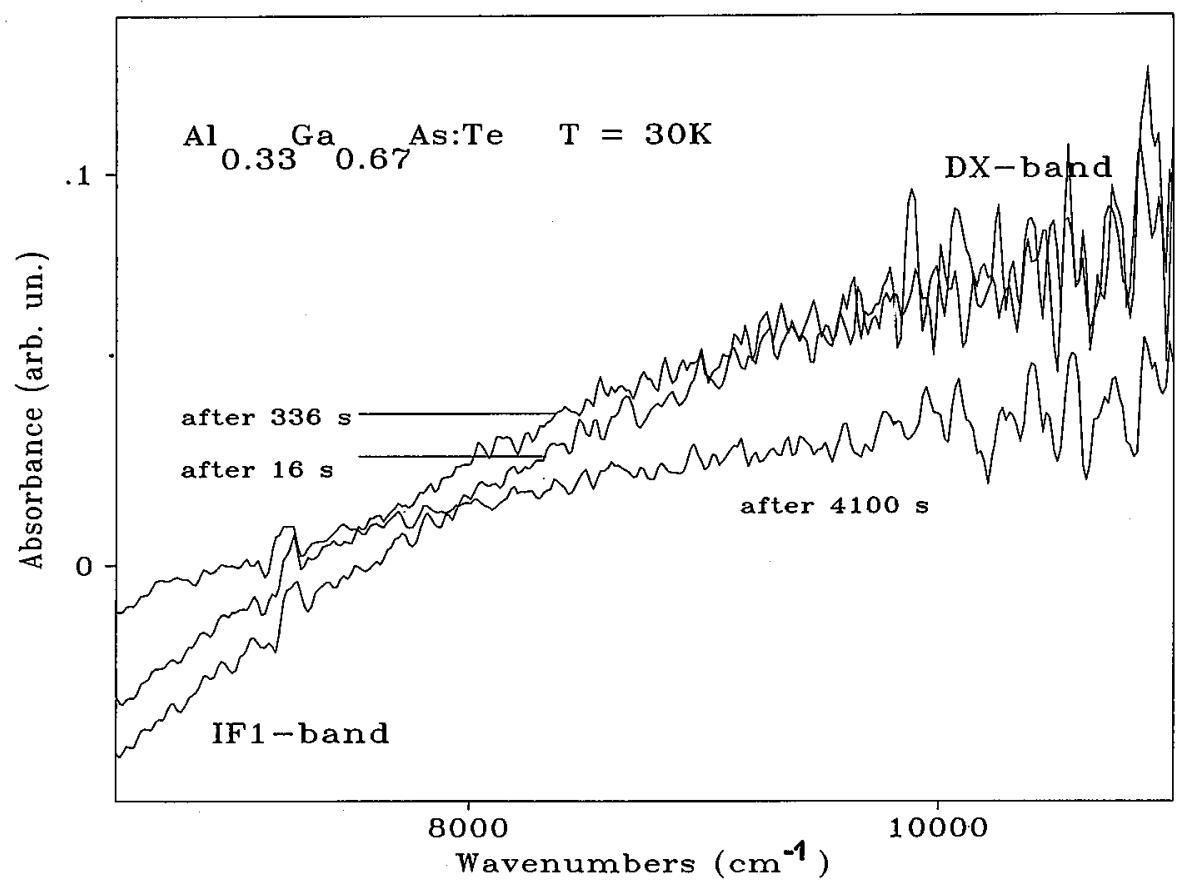

Fig. 1. The DX center photoionisation absorption merging with the absorption at the IF 1 band for different values of illumination time. Absorption spectra were recorded in equal time intervals. The spectrum registered after complete bleaching of the DX center absorption was taken as the reference. Therefore, the quantity measured is the defect absorbance: $\alpha_{\text {defect }} \cdot d$ (absorption coefficient originating from defect photoionisation, $\alpha_{\mathrm{DX}}$ or photopopulation, $\alpha_{\mathrm{IF} 1}$, times sample thïckness) vs. wa ve number.

$x \geq 0.33$ ) with an absorption band originating from a shallow donor to higher band minimum transition called IF1 band [5]. The absorption coefficient in the $1.2 \mathrm{eV}$. band scales with Te concentration and decreases during illumination due to photoionisation of the DX center. In the present experiment a low temperature (below $50 \mathrm{~K}$ ) and low light fluxes were used in order to slow down the photoionisation of the DX center. The DX center absorption is expected to decrease monotonically with the illumination time due to an irreversible transformation of the defect from the interstitial into a substitutional configuration [1]. But in fact, as we observe, at wave numbers greater than $c a .7500 \mathrm{~cm}^{-1}$ this absorption rises first to a certain value and only then decreases (Fig. 2).

This unusual behavior can only be explained by a population of an inter- 


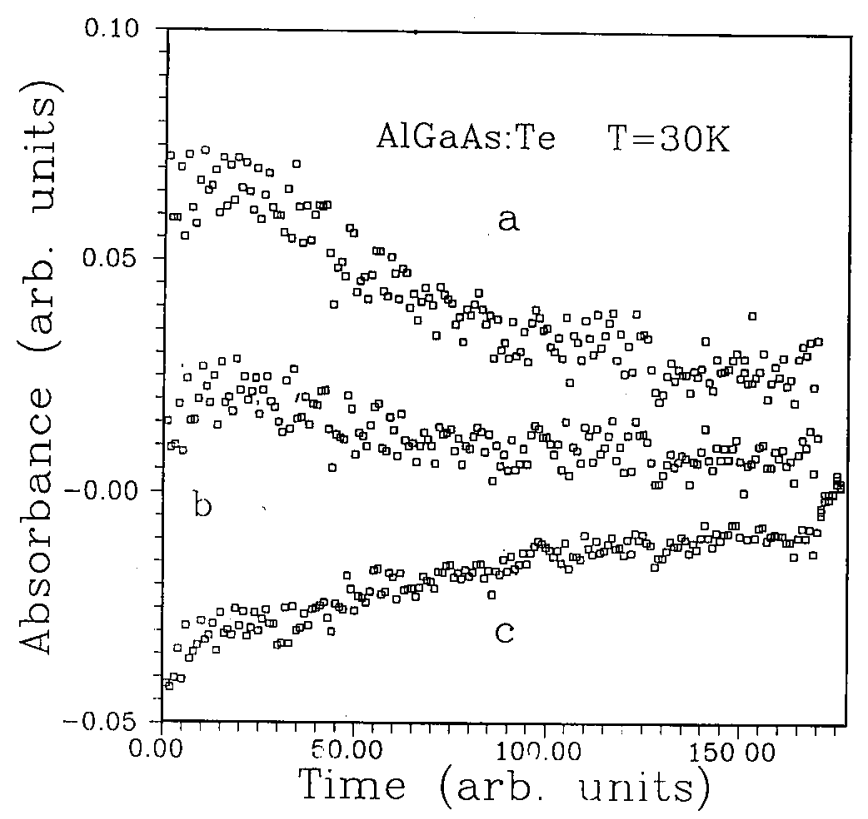

Fig. 2. Time evolution of the DX photoionisation absorption at chosen energies of the incident light - curve $a: \alpha(t)$ at $10000 \mathrm{~cm}^{-1}(1.2 \mathrm{eV})$, curve $b: \alpha(t)$ at $8000 \mathrm{~cm}^{-1}$ $(1 \mathrm{eV})$. For comparison the time dependence of the absorption at IF1 band, which is due to transition from the ground state of the bistable shallow donor (photopopulated during DX center photoionisation) to higher band minimum: curve $c\left(\alpha(t)\right.$ at $\left.6700 \mathrm{~cm}^{-1}\right)$.

mediate state, whose photoionisation cross-section is very similar to that of the DX center (the general line shape changes only a little as seen in Fig. 1). It is created due to photoionisation of DX center and its absorption vanishes for long illumination times. The latter means that the population of the new state is not only concominant with the DX photoionisation process and cannot be explained by photopopulation of an "DX-independent" state.

All above characteristics of the new state, as given above, i.e. instability, appearance during DX photoionisation and similar spectral dependence of the absorption coefficient (to that of the DX center), match very well with the properties of the DX intermediate charge state postulated from photocapacitance experiments [2].

The initial increase in DX absorption coefficient is observed in the whole wave number range (above $7500 \mathrm{~cm}^{-1}$ ) only for direct gap samples. For indirect band gap sample (with $x=0.41$ ) only a narrow band at $7800 \mathrm{~cm}^{-1}$ exhibits such behavior. Also these properties are in agreement with the results of the photocapacitance experiment [2]. We conclude therefore that anomalous properties of the kinetics of the DX photoionisation absorption, as observed in our experiment, can be taken as an indication for the participation of the intermediate charge state in the DX center photoionisation. 


\section{Acknowledgments}

We acknowledge many discussions with Dr. L. Dobaczewski and Dr. M. Godlewski.

\section{References}

[1] D.J. Chadi, K.J. Chang, Phys. Rev. Lett. 61, 873 (1988) and also T.N. Morgan, in: Proc. 15th Int. Conf. Def. Semicond., Budapest 1988, Ed. G. Ferenczi, Mater. Sci. Forum, Vol. 38-41, Trans Tech Publ., Switzerland 1989, p. 1079.

[2] L. Dobaczewski, P. Kaczor, Phys. Rev. Lett. 66, 68 (1991).

[3] L. Dobaczewski, P. Kaczor, M. Missous, I. Poole, A.R. Peaker, Z.R. Żytkiewicz, Phys. Rev. Lett. 68, 2508 (1992).

[4] Z.R. Żytkiewicz, S. Miotkowska, J. Cryst. Growth 121, 457 (1992).

[5] P. Kaczor, Z.R. Żytkiewicz, Acta Phys. Pol. A 80, 397 (1991); or P. Kaczor, Z.R. Żytkiewicz, to be published.

[6] D.V. Lang, R.A. Logan, Phys. Rev. Lett. 39, 635 (1977). 